\title{
Comparative clinical outcomes between direct oral anticoagulants and warfarin among elderly patients with non-valvular atrial fibrillation in the CMS medicare population
}

\author{
Alpesh Amin ${ }^{1} \cdot$ Allison Keshishian ${ }^{2}$. Oluwaseyi Dina ${ }^{3} \cdot$ Amol Dhamane $^{4} \cdot$ Anagha Nadkarni $^{4} \cdot$ Eric Carda $^{3}$. \\ Cristina Russ $^{3} \cdot$ Lisa Rosenblatt ${ }^{4} \cdot$ Jack Mardekian $^{3} \cdot$ Huseyin Yuce $^{5} \cdot$ Christine L. Baker $^{3}$
}

Published online: 28 March 2019

(C) The Author(s) 2019

\begin{abstract}
Atrial fibrillation (AF) prevalence increases with age; $>80 \%$ of US adults with AF are aged $\geq 65$ years. Compare the risk of stroke/systemic embolism (SE), major bleeding (MB), net clinical outcome (NCO), and major adverse cardiac events (MACE) among elderly non-valvular AF (NVAF) Medicare patients prescribed direct oral anticoagulants (DOACs) vs warfarin. NVAF patients aged $\geq 65$ years who initiated DOACs (apixaban, dabigatran, and rivaroxaban) or warfarin were selected from 01JAN2013-31DEC2015 in CMS Medicare data. Propensity score matching was used to balance DOAC and warfarin cohorts. Cox proportional hazards models estimated the risk of stroke/SE, MB, NCO, and MACE. 37,525 apixaban-warfarin, 18,131 dabigatran-warfarin, and 55,359 rivaroxaban-warfarin pairs were included. Compared to warfarin, apixaban (HR: 0.69; 95\% CI 0.59-0.81) and rivaroxaban (HR: 0.82; 95\% CI 0.73-0.91) had lower risk of stroke/SE, and dabigatran (HR: 0.88; 95\% CI 0.72-1.07) had similar risk of stroke/SE. Apixaban (MB: HR: 0.61; 95\% CI 0.57-0.67; NCO: HR: 0.64; 95\% CI 0.60-0.69) and dabigatran (MB: HR: 0.79; 95\% CI 0.71-0.89; NCO: HR: 0.84; 95\% CI 0.76-0.93) had lower risk of $\mathrm{MB}$ and $\mathrm{NCO}$, and rivaroxaban had higher risk of MB (HR: 1.08; 95\% CI 1.02-1.14) and similar risk of NCO (HR: 1.04; 95\% CI 0.99-1.09). Compared to warfarin, apixaban had a lower risk for stroke/SE, MB, and NCO; dabigatran had a lower risk of MB and NCO; and rivaroxaban had a lower risk of stroke/SE but higher risk of MB. All DOACs had lower risk of MACE compared to warfarin.
\end{abstract}

Keywords Apixaban · Dabigatran · Rivaroxaban · Warfarin · Non-valvular atrial fibrillation · Medicare

\section{Highlights}

Electronic supplementary material The online version of this article (https://doi.org/10.1007/s11239-019-01838-5) contains supplementary material, which is available to authorized users.

\section{Alpesh Amin}

anamin@uci.edu

Christine L. Baker

Christine.L.Baker@pfizer.com

1 Department of Medicine, University of California, 101 The City Drive South, Building 26, Room 1000, ZC-4076H, Orange, CA 92868, USA

2 STATinMED, Ann Arbor, MI, USA

3 Pfizer Inc., New York, NY, USA

4 Bristol-Myers Squibb Company, Lawrenceville, NJ, USA

5 New York City College of Technology, City University of New York, New York, NY, USA
- The prevalence of NVAF and risk of stroke increase with age.

- Few studies have compared DOACs to warfarin among elderly NVAF patients regarding such outcomes.

- This study showed that compared to warfarin, all DOACs were associated with lower risk of MACE, and there were varying rates of stroke/SE, MB, and $\mathrm{NCO}$ between the individual DOACs and warfarin.

- The findings warrant more studies to better understand effectiveness and safety profiles in the elderly NVAF population. 


\section{Introduction}

The 2010 Global Burden of Disease Study estimated the worldwide age-adjusted prevalence of atrial fibrillation (AF) at 596 per 100,000 men and 373 per 100,000 women, equating to 33.5 million individuals (20.9 and 12.6 million men and women, respectively) [1]. In the United States, the estimated prevalence of AF is $3-5$ million [2, $3]$. The proportion of AF patients was found to increase sharply with age, especially in people aged $\geq 65$ years, who account for three-quarters of the AF population [3].

Patients with AF diagnoses are at a nearly fivefold greater risk of stroke [4]. Moreover, the AF-attributable risk for ischemic stroke is age-dependent and increases from 4.6 to $7.9 \%$ to $>10 \%$ among patients aged $50-59$, 60-69, and $\geq 70$ years, respectively [4]. Hence, the stroke risk stratification schema $\mathrm{CHA}_{2} \mathrm{DS}_{2}$-VASc score considers older age (65-74 and $\geq 75$ years) as a risk factor for stroke and thromboembolism in AF patients [5].

Oral anticoagulants (OACs) prevent stroke and systemic embolism (SE) among AF patients; they are recommended by the American College of Cardiology (ACC) and the American Heart Association (AHA) guidelines for patients with non-valvular AF (NVAF) and prior stroke, transient ischemic attack (TIA), or a $\mathrm{CHA}_{2} \mathrm{DS}_{2}$-VASc score $\geq 2$ [6]. Warfarin, a vitamin $\mathrm{K}$ antagonist (VKA), has been used for stroke prevention among AF patients for decades. However, the narrow therapeutic window and increased risk of bleeding have hindered use, especially among the elderly [6].

In recent years, randomized clinical trials have demonstrated that compared to warfarin, direct OACs (DOACs) - including apixaban, dabigatran, edoxaban, and rivaroxaban - were all associated with similar to lower risk of stroke/SE and major bleeding (MB) among elderly patients [7-9]. Introduced in 2008, the Fit-fOR-The-Aged (FORTA) classification is the first system with both negative (harmful or critical drugs: D and C labels) and positive (beneficial drugs: A and B labels) labelling at the individual drug and drug group levels. Based on FORTA and the Delphi process, warfarin, dabigatran, edoxaban, and rivaroxaban were labelled B (beneficial; safely and effectively treat AF), and apixaban was labeled A (absolutely; most beneficial risk-benefit ratio) for the treatment of $\mathrm{AF}$ patients aged $>65$ years [10].

Using the largest US claims database of elderly patients, we evaluated real-world comparative risks of stroke/SE, $\mathrm{MB}$, net clinical outcomes (stroke/SE or MB [NCO]), and major adverse cardiac events (MACE) among NVAF patients who initiated either DOACs (apixaban, dabigatran, and rivaroxaban) or warfarin. This study added more recent data and additional outcome measures to our previous study, which provides comprehensive and current evidence to help prevent stroke among the elderly NVAF population [11]. The results also supplement clinical trials and add key information to real-world literature.

\section{Methods}

\section{Data source}

This retrospective observational study used the fee-for-service (FFS) US Centers for Medicare \& Medicaid Services (CMS) data from 01JAN2012-31DEC2015. This dataset is composed of adults aged $\geq 65$ years, certain young people with disabilities, and people with end-stage renal disease. As of 2015, > 38 million beneficiaries were enrolled in this insurance [12]. The data include institutional (inpatient, skilled nursing facility, home health, hospice, and hospital outpatient) and non-institutional (physician/supplier-carrier and durable medical equipment) claims and Part $\mathrm{D}$ prescription claims, coded using International Classification of Diseases, Ninth/Tenth Revision, Clinical Modification (ICD9/10-CM) diagnosis and procedure codes, the Health Care Common Procedure Coding System, Current Procedural Terminology codes, and National Drug Codes [13].

\section{Patient selection}

AF (ICD-9-CM: 427.31 or ICD-10-CM: I48.0-I48.2, I48.91) patients aged $\geq 65$ years with $\geq 1$ pharmacy claim for apixaban, dabigatran, edoxaban, rivaroxaban, or warfarin between 01JAN2013-31DEC2015 (identification period) were selected. The first DOAC claim date during the identification period was designated as the index date for patients with any DOAC claim; the first warfarin prescription date was designated as the index date for those without a DOAC claim [14]. Patients were also required to have continuous health plan enrollment with both medical and pharmacy benefits for the 12-month pre-index (baseline) period.

To select OAC treatment-naïve patients, those with any OAC claim during the baseline period were excluded. Patients with evidence of valvular heart disease or transient AF during the baseline period were also excluded. To omit OAC use for the treatment or prophylaxis of venous thromboembolism (VTE), patients with VTE in the baseline period or who had hip or knee replacement surgery within 6 weeks prior to the index date were excluded. Detailed selection criteria appear in Fig. 1.

\section{Outcome measures}

The primary outcomes were the occurrence of stroke/SE and $\mathrm{MB}$, identified by hospitalizations with stroke/SE or MB as 
Fig. 1 Patient selection criteria

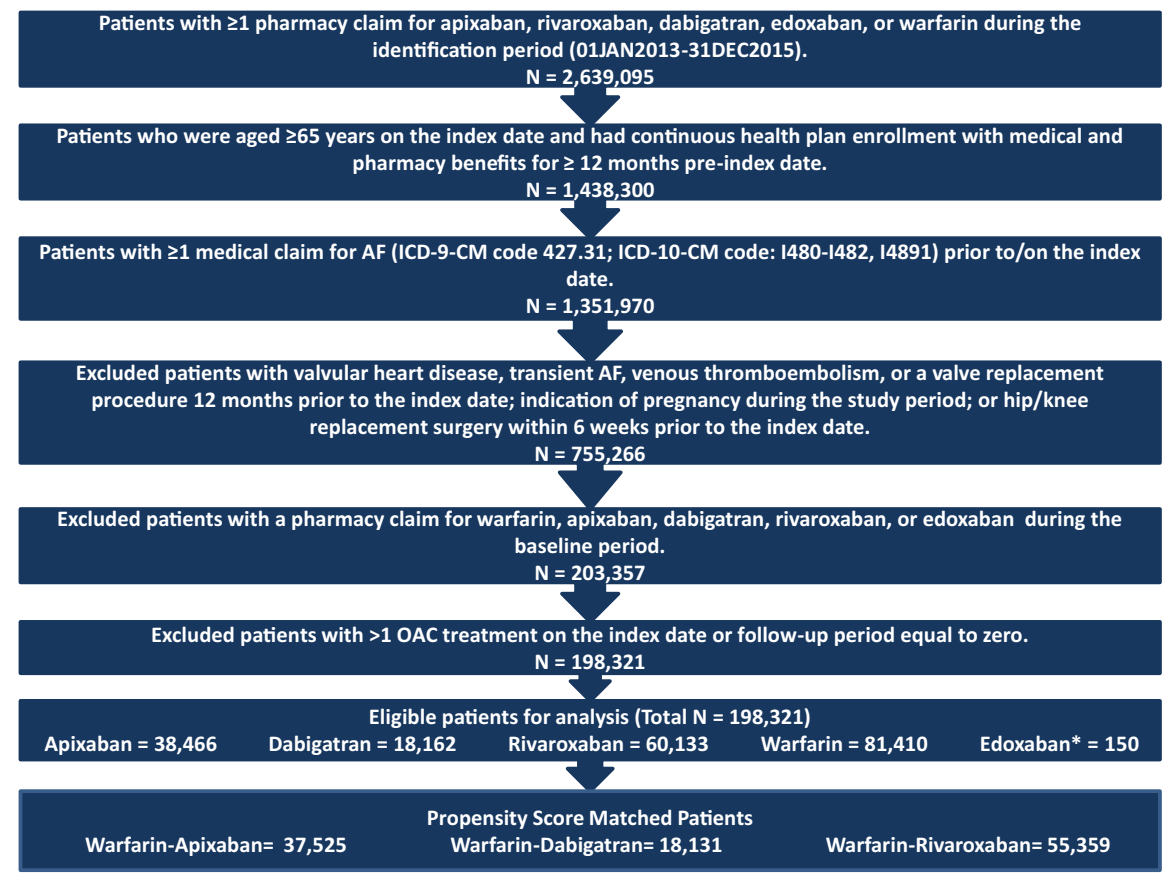

*Edoxaban was not included in the analysis given the small sample size.

AF: atrial fibrillation; ICD-9-CM: International Classification of Diseases, Ninth Revision, Clinical Modification;

ICD-10-CM: International Classification of Diseases, Tenth Revision, Clinical Modification; OAC: oral anticoagulant.

the principal diagnosis. Stroke/SE was further categorized by ischemic stroke, hemorrhagic stroke, and SE; MB was categorized by gastrointestinal (GI) bleeding, intracranial hemorrhage (ICH), and MB at other key sites $[15,16]$.

The secondary outcomes were NCO (a composite of stroke/SE and MB) and MACE, comprised of stroke (hemorrhagic and ischemic stroke), myocardial infarction (MI), and all-cause death. Claims databases cannot evaluate cardiovascular-related death, so the MACE definition included all-cause death.

Patients were censored at the earliest of the discontinuation date of the index treatment (no evidence of a prescription for 30 days from the last day of the index medication days' of supply), date of switch from the index drug to another OAC (a prescription for an OAC other than the index drug within 30 days before or after the discontinuation date), date of death, end of continuous enrollment, or end of study.

\section{Statistical methods}

One-to-one propensity score matching (PSM) was conducted between DOACs and warfarin (apixaban versus warfarin, dabigatran versus warfarin, and rivaroxaban versus warfarin) to control for potential confounders such as baseline demographics and clinical characteristics.

Using established methodology, propensity scores were generated by logistic regression. Age, sex, US geographic region, Charlson comorbidity index (CCI) [17], $\mathrm{CHA}_{2} \mathrm{DS}_{2}$-VASc, and HAS-BLED scores, prior bleeding and stroke, comorbidities, baseline co-medications, and baseline inpatient visits were included in the models as covariates. The nearest neighbor without replacement method and a caliper of 0.01 were implemented in the PSM [18]. After PSM, the balance of covariates was checked based on standardized differences, with a threshold of $10 \%$ [19].

For post-PSM cohorts, the incidence of primary and secondary outcomes was calculated as the number of events per 100 person-years.

Cox proportional hazards models with robust sandwich estimates were used to evaluate the hazard ratios (HRs) of stroke/SE, MB, NCO, and MACE in each matched cohort [18]. After ensuring all the matched baseline covariates were balanced post-PSM, OAC treatment was included in the Cox models as the only independent variable.

Sensitivity analysis was conducted wherein patients were censored at 6 months of follow-up, creating more balance between cohorts.

Statistical analyses were performed using the Statistical Analysis System (SAS) Version 9.3 (Cary, NC).

\section{Results}

The study included eligible 198,171 patients; $81,410(41.1 \%)$ were prescribed warfarin, 38,466 (19.4\%) apixaban, 18,162 (9.2\%) dabigatran, and 60,133 (30.3\%) rivaroxaban (Fig. 1). Edoxaban was excluded due to small sample size $(\mathrm{N}=150)$. Before PSM, patients who initiated warfarin were older with 
a mean age of 79 years, followed by those who initiated apixaban (78 years), rivaroxaban (78 years), and dabigatran (77 years). In addition, warfarin patients also had higher CCI and $\mathrm{CHA}_{2} \mathrm{DS}_{2}$-VASc scores than DOAC patients (Table 1).

Through PSM, 37,525 apixaban, 18,131 dabigatran, and 55,359 rivaroxaban patients were separately matched to warfarin patients. Baseline characteristics were balanced after matching with mean standardized differences $<10 \%$. For the matched cohorts, the means were: age: 77-78 years, $\mathrm{CHA}_{2} \mathrm{DS}_{2}$-VASc scores: 4.4-4.6, and HAS-BLED scores: 3.2-3.4 (Table 2). Patient data were assessed for a mean duration of $8-10$ months. $71 \%, 80 \%$, and $66 \%$ of patients were prescribed the standard dose of DOAC (apixaban $5 \mathrm{mg}$, dabigatran $150 \mathrm{mg}$, and rivaroxaban $20 \mathrm{mg}$ ), respectively.

\section{Stroke/SE and MB}

Compared to warfarin, apixaban (HR: 0.69; 95\% confidence interval [CI] 0.59-0.81, $\mathrm{p}<0.001)$ and rivaroxaban (HR: 0.82 ; 95\% CI $0.73-0.91, \mathrm{p}<0.001)$ were associated with a significantly lower risk of stroke/SE; dabigatran (HR: 0.88; 95\% CI $0.72-1.07, \mathrm{p}=0.206$ ) was associated with a nonsignificantly lower risk of stroke/SE (Fig. 2). All DOACs were associated with a lower risk of hemorrhagic stroke versus warfarin.

Compared to warfarin, apixaban (HR: 0.61; 95\% CI 0.57-0.67, p<0.001), and dabigatran (HR: 0.79; 95\% CI $0.71-0.89, \mathrm{p}<0.001$ ) were associated with a significantly lower risk of $\mathrm{MB}$, and rivaroxaban (HR: 1.08 ; 95\% CI $1.02-1.14, \mathrm{p}=0.006$ ) was associated with a higher risk of MB, mainly due to GI bleeding (Fig. 2). All DOACs were associated with a lower risk of ICH versus warfarin.

\section{NCO and MACE}

As a composite of stroke/SE and MB, the risk of NCO was significantly lower than warfarin for apixaban (HR: 0.64; 95\% CI 0.60-0.69, p <0.001) and dabigatran, (HR: 0.84; 95\% CI $0.76-0.93, \mathrm{p}=0.001)$ but similar for rivaroxaban (HR: 1.04; 95\% CI 0.99-1.09, $\mathrm{p}=0.169$ ) (Fig. 3).

Compared to warfarin, all DOACs were associated with a lower risk of MACE (apixaban: HR: $0.70 ; 95 \%$ CI $0.67-0.74, \mathrm{p}<0.001$; dabigatran: HR: 0.76 ; $95 \%$ CI $0.71-0.82, \mathrm{p}<0.001$; rivaroxaban: HR: $0.83 ; 95 \% \mathrm{CI}$ $0.80-0.86$, p $<0.001$; Fig. 3$)$.

\section{Sensitivity analysis}

In the sensitivity analysis wherein the follow-up period was censored at 6 months, the results were consistent with the main analysis (Supplemental Table 1).

\section{Discussion}

Using Medicare FFS data from 2012 to 2015, this study showed that compared to warfarin among elderly patients with NVAF, apixaban was associated with significant lower risks of stroke/SE, MB, NCO, and MACE. Dabigatran was associated with significantly lower risks of $\mathrm{MB}$, NCO, and MACE as well as a numerically lower risk of stroke/SE. Rivaroxaban was associated with lower risks of stroke/SE and MACE, but higher MB and numerically higher NCO risks compared to warfarin.

The study results supplement RCT findings for apixaban, dabigatran, and rivaroxaban compared to warfarin and their corresponding age subgroup analyses [20-25]. In the RE-LY trial, patients (overall and $\geq 75$ years) with $150 \mathrm{mg}$ dabigatran had lower rates of stroke/SE and similar rates of MB compared to warfarin [20,23]. In this real-world study among NVAF patients aged $\geq 65$ years, $150 \mathrm{mg}$ and $75 \mathrm{mg}$ dabigatran showed numerically lower stroke/SE and significantly lower MB risks versus warfarin. Although NCO was not studied in the RE-LY trial's elderly group, overall dabigatran and warfarin patient analysis demonstrated that compared to warfarin, $150 \mathrm{mg}$ twice-daily dabigatran was associated with a non-significantly lower risk of net clinical benefit (a composite of stroke/SE, pulmonary embolism, MI, death, and MB) [20]. In this study, elderly dabigatran patients were associated with significantly lower NCO and MACE risks than warfarin patients.

In the ARISTOTLE trial, apixaban was associated with lower rates of stroke/SE, MB, and net clinical events (stroke/SE, MB, and all-cause death) compared to warfarin among all patients and patients aged $\geq 65$ years $[22,25]$. This study found consistent trends. In the ROCKET AF trial, rivaroxaban was associated with a non-inferior rate of stroke/SE and similar rate of MB compared to warfarin [21]. Among patients aged $\geq 75$ years, 20 and $15 \mathrm{mg}$ daily rivaroxaban showed a numerically lower risk of stroke/SE but a higher risk of MB compared to warfarin [24]. This study found similar trends between rivaroxaban and warfarin among patients aged $\geq 65$ years. To the best of our knowledge, no previous studies have compared net clinical benefits between rivaroxaban and warfarin.

Several real-world studies have focused on effectiveness and safety comparisons between DOACs and warfarin in an elderly NVAF population [11, 26-29]. Our previous study of the elderly Medicare population from 2012 to 2014 consistent results of stroke/SE and major bleeding were found for the comparisons between DOACs and warfarin [11]. This study provides more recent and comprehensive analysis with updated data and added NCO and MACE outcomes. Using Medicare data from 2010 to 2012, Graham et al. [26] demonstrated that 
Table 1 Baseline descriptive table before PSM

\begin{tabular}{|c|c|c|c|c|c|c|c|c|c|c|c|}
\hline & \multicolumn{2}{|c|}{$\begin{array}{l}\text { Warfarin } \\
(\mathrm{N}=81,410)\end{array}$} & \multicolumn{3}{|c|}{ Apixaban $(\mathrm{N}=38,466)$} & \multicolumn{3}{|c|}{ Dabigatran $(\mathrm{N}=18,162)$} & \multicolumn{3}{|c|}{ Rivaroxaban $(\mathrm{N}=60,133)$} \\
\hline & N/mean & $\% / \mathrm{SD}$ & N/mean & $\% / \mathrm{SD}$ & $\mathrm{STD}^{\mathrm{a}}$ & N/mean & $\% / \mathrm{SD}$ & $\mathrm{STD}^{\mathrm{a}}$ & $\mathrm{N} /$ mean & $\% / \mathrm{SD}$ & $\mathrm{STD}^{\mathrm{a}}$ \\
\hline Age & 78.9 & 7.5 & 78.3 & 7.5 & 6.9 & 77.0 & 7.0 & 25.0 & 77.6 & 7.3 & 16.8 \\
\hline $65-74$ & 26,091 & $32.0 \%$ & 13,627 & $35.4 \%$ & 7.1 & 7479 & $41.2 \%$ & 19.0 & 23,255 & $38.7 \%$ & 13.9 \\
\hline $75-84$ & 35,012 & $43.0 \%$ & 15,916 & $41.4 \%$ & 3.3 & 7607 & $41.9 \%$ & 2.3 & 25,119 & $41.8 \%$ & 2.5 \\
\hline$\geq 85$ & 20,307 & $24.9 \%$ & 8923 & $23.2 \%$ & 4.1 & 3076 & $16.9 \%$ & 19.8 & 11,759 & $19.6 \%$ & 13.0 \\
\hline \multicolumn{12}{|l|}{$\operatorname{Sex}$} \\
\hline Male & 41,002 & $50.4 \%$ & 18,581 & $48.3 \%$ & 4.1 & 9338 & $51.4 \%$ & 2.1 & 29,894 & $49.7 \%$ & 1.3 \\
\hline Female & 40,408 & $49.6 \%$ & 19,885 & $51.7 \%$ & 4.1 & 8824 & $48.6 \%$ & 2.1 & 30,239 & $50.3 \%$ & 1.3 \\
\hline \multicolumn{12}{|l|}{ Race } \\
\hline White & 73,714 & $90.5 \%$ & 35,311 & $91.8 \%$ & 4.4 & 16,309 & $89.8 \%$ & 2.5 & 54,642 & $90.9 \%$ & 1.1 \\
\hline Black & 4246 & $5.2 \%$ & 1432 & $3.7 \%$ & 7.2 & 785 & $4.3 \%$ & 4.2 & 2336 & $3.9 \%$ & 6.4 \\
\hline Hispanic & 1037 & $1.3 \%$ & 417 & $1.1 \%$ & 1.8 & 290 & $1.6 \%$ & 2.7 & 931 & $1.5 \%$ & 2.3 \\
\hline Other & 2413 & $3.0 \%$ & 1306 & $3.4 \%$ & 2.5 & 778 & $4.3 \%$ & 7.1 & 2224 & $3.7 \%$ & 4.1 \\
\hline \multicolumn{12}{|l|}{ Geographic region } \\
\hline Northeast & 16,018 & $19.7 \%$ & 6514 & $16.9 \%$ & 7.1 & 3606 & $19.9 \%$ & 0.4 & 10,596 & $17.6 \%$ & 5.3 \\
\hline North Central & 25,076 & $30.8 \%$ & 7911 & $20.6 \%$ & 23.6 & 4184 & $23.0 \%$ & 17.6 & 13,341 & $22.2 \%$ & 19.6 \\
\hline South & 26,486 & $32.5 \%$ & 17,229 & $44.8 \%$ & 25.4 & 6953 & $38.3 \%$ & 12.0 & 25,007 & $41.6 \%$ & 18.8 \\
\hline West & 13,745 & $16.9 \%$ & 6791 & $17.7 \%$ & 2.0 & 3387 & $18.6 \%$ & 4.6 & 11,080 & $18.4 \%$ & 4.0 \\
\hline Other & 85 & $0.1 \%$ & 21 & $0.1 \%$ & 1.8 & 32 & $0.2 \%$ & 1.9 & 109 & $0.2 \%$ & 2.0 \\
\hline Medicaid dual-eligibility & 18,908 & $23.2 \%$ & 7488 & $19.5 \%$ & 9.2 & 4268 & $23.5 \%$ & 0.6 & 13,100 & $21.8 \%$ & 3.5 \\
\hline Part D low-income subsidy & 21,374 & $26.3 \%$ & 8560 & $22.3 \%$ & 9.3 & 4814 & $26.51 \%$ & 0.6 & 14,734 & $24.5 \%$ & 4.0 \\
\hline \multicolumn{12}{|l|}{ Baseline comorbidity } \\
\hline Deyo-Charlson comorbidity index & 3.1 & 2.8 & 2.9 & 2.6 & 9.4 & 2.5 & 2.4 & 24.7 & 2.7 & 2.5 & 17.9 \\
\hline CHADS2 score & 2.9 & 1.4 & 2.8 & 1.5 & 6.9 & 2.6 & 1.4 & 19.1 & 2.7 & 1.4 & 15.3 \\
\hline $\mathrm{CHA}_{2} \mathrm{DS}_{2}$-VASc score & 4.7 & 1.7 & 4.6 & 1.8 & 5.5 & 4.4 & 1.7 & 20.2 & 4.5 & 1.7 & 14.3 \\
\hline HAS-BLED score ${ }^{\mathrm{b}}$ & 3.3 & 1.3 & 3.4 & 1.3 & 4.3 & 3.2 & 1.2 & 14.1 & 3.3 & 1.2 & 5.2 \\
\hline Baseline prior bleed & 24,780 & $30.4 \%$ & 11,807 & $30.7 \%$ & 0.6 & 4731 & $26.0 \%$ & 9.8 & 17,374 & $28.9 \%$ & 3.4 \\
\hline Baseline prior stroke & 12,496 & $15.3 \%$ & 5280 & $13.7 \%$ & 4.6 & 2159 & $11.9 \%$ & 10.1 & 7385 & $12.3 \%$ & 8.9 \\
\hline Congestive heart failure & 29,326 & $36.0 \%$ & 12,064 & $31.4 \%$ & 9.9 & 5118 & $28.2 \%$ & 16.9 & 17,287 & $28.7 \%$ & 15.6 \\
\hline Diabetes & 32,705 & $40.2 \%$ & 13,602 & $35.4 \%$ & 9.9 & 6737 & $37.1 \%$ & 6.3 & 21,456 & $35.7 \%$ & 9.3 \\
\hline Hypertension & 71,416 & $87.7 \%$ & 34,649 & $90.1 \%$ & 7.5 & 15,964 & $87.9 \%$ & 0.5 & 53,191 & $88.5 \%$ & 2.3 \\
\hline Renal disease & 21,021 & $25.8 \%$ & 8599 & $22.4 \%$ & 8.1 & 2892 & $15.9 \%$ & 24.5 & 10,465 & $17.4 \%$ & 20.6 \\
\hline Myocardial infarction & 12,024 & $14.8 \%$ & 5040 & $13.1 \%$ & 4.8 & 1940 & $10.7 \%$ & 12.3 & 7224 & $12.0 \%$ & 8.1 \\
\hline Dyspepsia or stomach discomfort & 17,317 & $21.3 \%$ & 8699 & $22.6 \%$ & 3.2 & 3607 & $19.9 \%$ & 3.5 & 13,060 & $21.7 \%$ & 1.1 \\
\hline Peripheral vascular disease & 46,697 & $57.4 \%$ & 22,742 & $59.1 \%$ & 3.6 & 9689 & $53.3 \%$ & 8.1 & 33,670 & $56.0 \%$ & 2.8 \\
\hline Peripheral artery disease & 20,131 & $24.7 \%$ & 8932 & $23.2 \%$ & 3.5 & 3635 & $20.0 \%$ & 11.3 & 13,237 & $22.0 \%$ & 6.4 \\
\hline Transient ischemic attack & 6411 & $7.9 \%$ & 3528 & $9.2 \%$ & 4.6 & 1342 & $7.4 \%$ & 1.8 & 4751 & $7.9 \%$ & 0.1 \\
\hline Coronary artery disease & 40,079 & $49.2 \%$ & 19,962 & $51.9 \%$ & 5.3 & 8367 & $46.1 \%$ & 6.3 & 29,066 & $48.3 \%$ & 1.8 \\
\hline \multicolumn{12}{|l|}{ Baseline medication use } \\
\hline Angiotensin converting enzyme inhibitor & 30,102 & $37.0 \%$ & 13,194 & $34.3 \%$ & 5.6 & 6875 & $37.9 \%$ & 1.8 & 21,463 & $35.7 \%$ & 2.7 \\
\hline Amiodarone & 5612 & $6.9 \%$ & 4300 & $11.2 \%$ & 15.0 & 1636 & $9.0 \%$ & 7.8 & 5308 & $8.8 \%$ & 7.2 \\
\hline Angiotensin receptor blocker & 17,030 & $20.9 \%$ & 10,056 & $26.1 \%$ & 12.3 & 4498 & $24.8 \%$ & 9.2 & 15,149 & $25.2 \%$ & 10.2 \\
\hline Beta blockers & 42,053 & $51.7 \%$ & 22,070 & $57.4 \%$ & 11.5 & 9756 & $53.7 \%$ & 4.1 & 32,812 & $54.6 \%$ & 5.8 \\
\hline $\mathrm{H} 2$-receptor antagonist & 5699 & $7.0 \%$ & 2828 & $7.4 \%$ & 1.4 & 1214 & $6.7 \%$ & 1.3 & 4181 & $7.0 \%$ & 0.2 \\
\hline Proton pump inhibitor & 24,020 & $29.5 \%$ & 13,008 & $33.8 \%$ & 9.3 & 5358 & $29.5 \%$ & 0.0 & 19,152 & $31.8 \%$ & 5.1 \\
\hline Anti-platelets & 15,589 & $19.1 \%$ & 9235 & $24.0 \%$ & 11.8 & 3450 & $19.0 \%$ & 0.4 & 13,101 & $21.8 \%$ & 6.5 \\
\hline Statins & 45,149 & $55.5 \%$ & 23,492 & $61.1 \%$ & 11.4 & 10,476 & $57.7 \%$ & 4.5 & 34,956 & $58.1 \%$ & 5.4 \\
\hline Inpatient admission & 36,572 & $44.9 \%$ & 15,168 & $39.4 \%$ & 11.1 & 6830 & $37.6 \%$ & 14.9 & 24,807 & $41.3 \%$ & 7.4 \\
\hline
\end{tabular}

Std Difference greater than 10 is considered significant is given in bolditalic

$\mathrm{CHA}_{2} \mathrm{DS}_{2}$-VASc: congestive heart failure, hypertension, age $\geq 75$ years, diabetes mellitus, prior stroke or transient ischemic attack or thromboembolism, vascular disease, age 65-74 years, sex category; HAS-BLED: hypertension, abnormal renal and liver function, stroke, bleeding, labile INRs (international normalized ratio), elderly, drugs, and alcohol; PSM: propensity score matching; SD: standard deviation

${ }^{\text {a }}$ Std Difference $=100 *$ lactual std diffl

${ }^{\mathrm{b}}$ As the INR value was not available in the data, a modified HAS-BLED score was calculated using a range of 0 to 8 


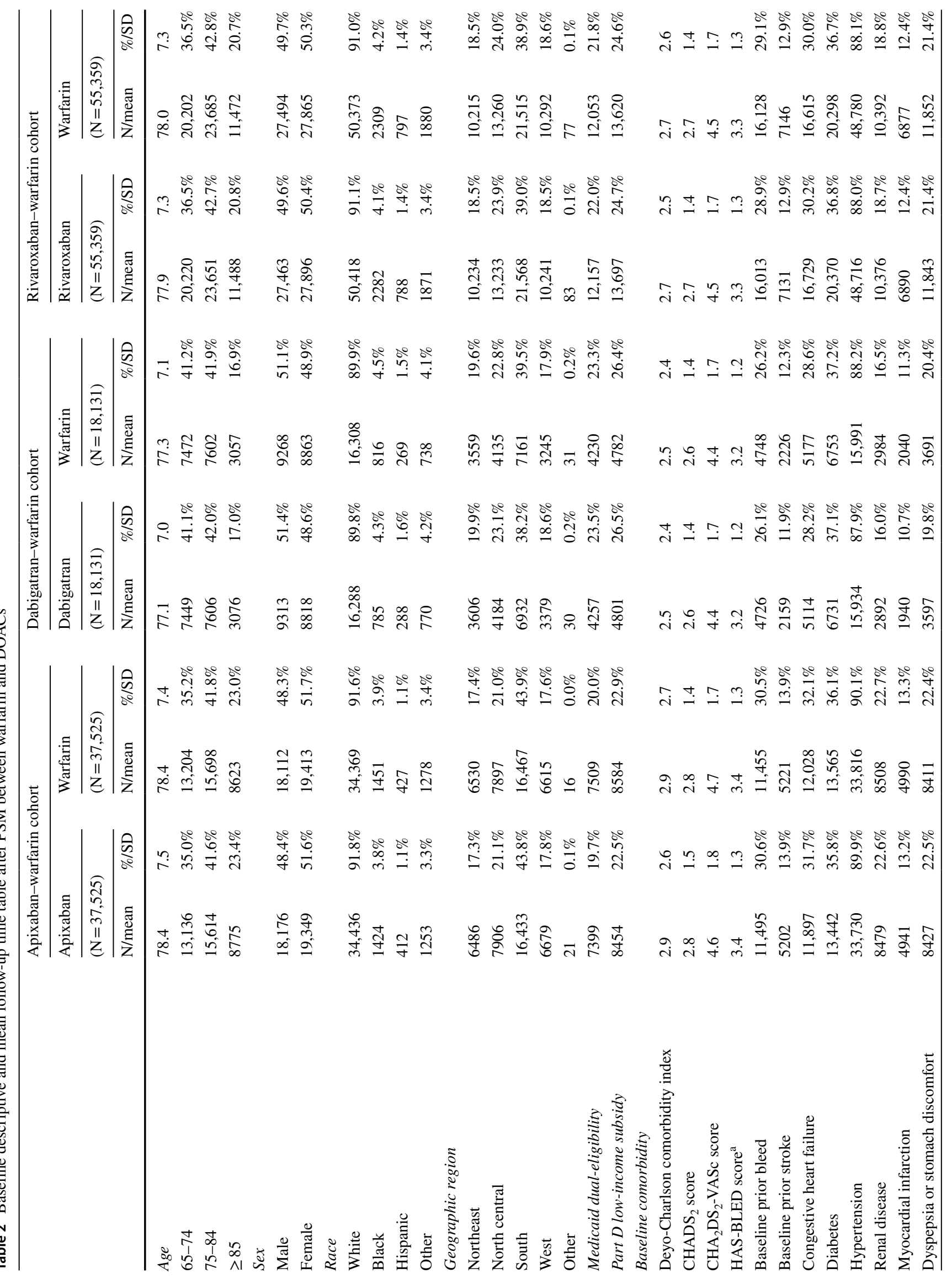




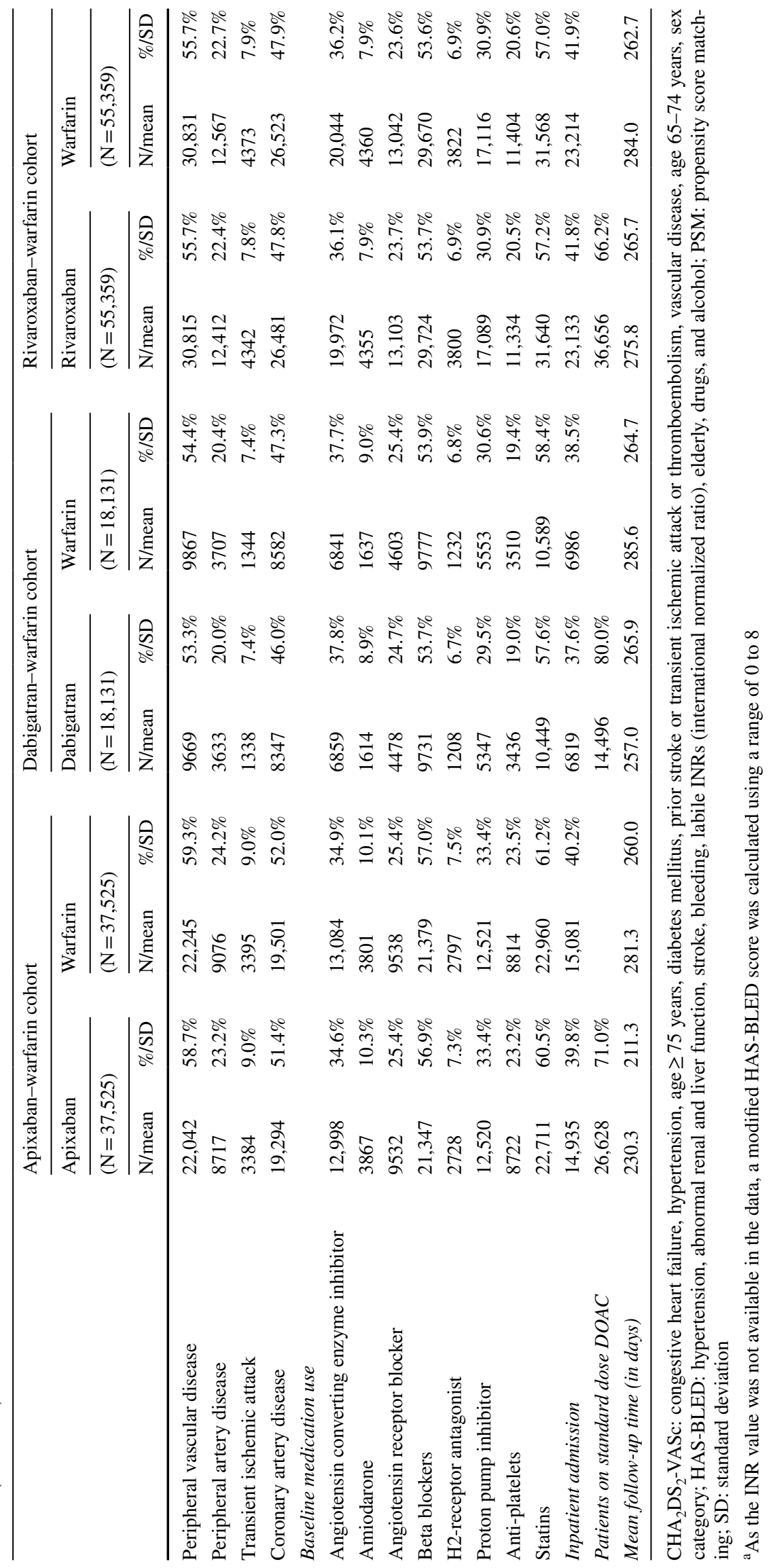




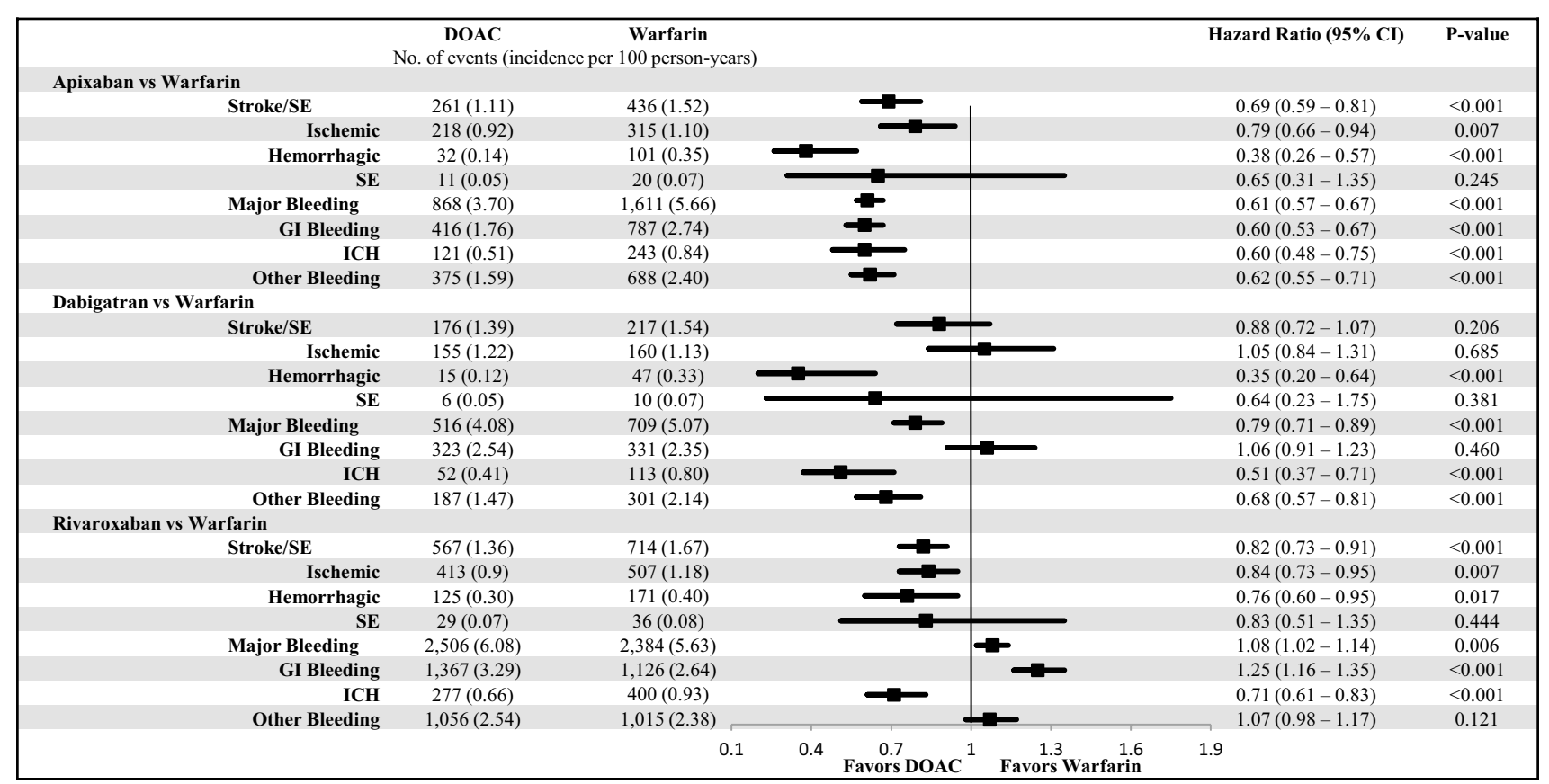

$\mathrm{CI}$ : confidence interval; DOAC: direct oral anticoagulant; GI: gastrointestinal; ICH: intracranial hemorrhage; SE: systemic embolism

Fig. 2 Incidence rate and hazard ratio of stroke/SE and major bleeding for propensity score-matched patients

compared to warfarin, elderly NVAF dabigatran initiators (aged $\geq 65$ years) were associated with lower risks of ischemic stroke, ICH, and death; similar risk of acute MI and $\mathrm{MB}$; and a higher major GI bleeding risk. Our results (over an updated time-frame) showed consistent trends for ICH and GI bleeding. However, the ischemic stroke risk was similar, and the MB risk was lower for dabigatran versus warfarin patients in our study. Using Humana data, Deitelzweig et al. [27] found that NVAF patients aged $\geq 65$ years with Medicare Advantage coverage who were treated with apixaban were associated with significantly lower risks of stroke/SE and MB compared to warfarin. This study noted consistent trends.

A few other real-world studies among DOACs and warfarin have provided comparative effectiveness and safety information by conducting subgroup analyses for age subgroups [30-32]. Using MarketScan and Optum data from 2010 to 2012, Seeger et al. showed that among patients aged 65-74 years, compared to warfarin, dabigatran was associated with similar risk for stroke and lower risk for MB; among those aged $\geq 75$ years, dabigatran was associated with lower risk for stroke and similar risk for MB [30]. Using the MarketScan data from 2010 to 2014, Norby et al. [31] found that among patients aged $\geq 75$ years, rivaroxaban was associated with a similar risk for ischemic stroke and MI, a lower ICH risk, and a higher GI bleeding risk compared to warfarin. Using a pooled dataset, $\mathrm{Li}$ et al. [32] demonstrated that among elderly patients, apixaban was associated with similar
(65-74) to lower $(\geq 75)$ stroke/SE risk and a lower (65-74 and $\geq 75$ ) MB risk compared to warfarin. The comparisons between DOACs and warfarin in our study showed trends generally consistent with previous literature. However, more studies are needed to better understand effectiveness and safety profiles in elderly populations. Moreover, as DOAC use increases, further research will be necessary to assist in decision-making for such populations [33].

Despite growing evidence of improved safety with DOACs, warfarin is still widely used in high-risk NVAF populations [34]. Our study provides a current and comprehensive analysis comparing DOACs and warfarin regarding the risk of stroke/SE, MB, NCOs, and MACE among elderly US Medicare NVAF patients. Given the distinct clinical characteristics of the elderly NVAF population, the study results may add useful information to the literature to assist in disease management decision making.

This study has several limitations. Given its observational nature, confounding factors may have impacted the results. To control for potential confounders, a comprehensive list of baseline covariates was included in the PSM, including patient demographics and clinical characteristics. However, variables such as over-the-counter use of aspirin, serum creatinine/creatinine clearance, and laboratory test result values are not captured in the Medicare data. As claims data analysis, the study may also be subject to coding errors and inaccurate or incomplete clinical information. For example, treatments recorded based on prescription claims include no 


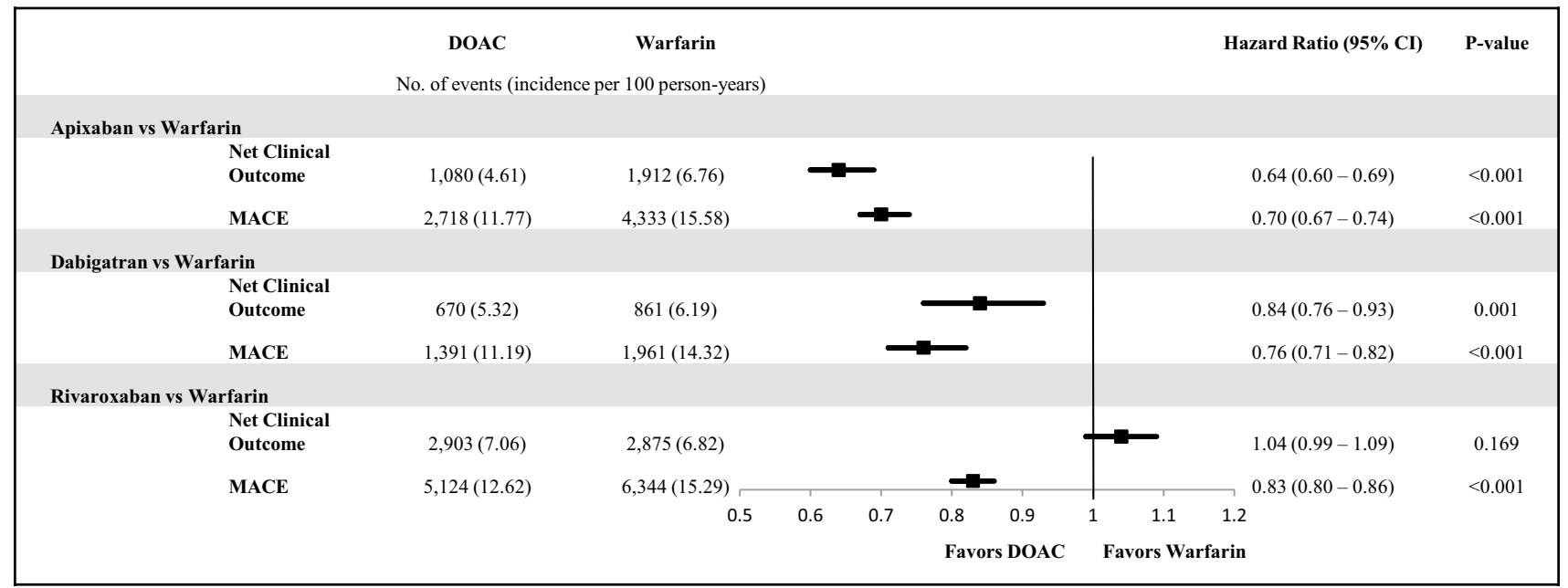

$\mathrm{Cl}$ : confidence interval; DOAC: direct oral anticoagulant; MACE: major adverse cardiac events

Fig. 3 Incidence rates and hazard ratios of net clinical outcome and MACE for propensity score-matched patients

evidence of drug adherence. Moreover, since international normalized ratio values were not obtained, the quality of warfarin treatment could not be evaluated and the calculation for HAS-BLED score was modified. Moreover, proper dosage for DOACs based on age, renal function, and weight could not be assessed.

In summary, in the elderly Medicare population with NVAF, compared to warfarin, the DOACs were associated with a lower to similar risk of stroke/SE and MACE, but with varying comparative risks for $\mathrm{MB}$ and $\mathrm{NCO}$.

Funding This work was funded by Pfizer Inc. and Bristol-Myers Squibb.

\section{Compliance with ethical standards}

Conflict of interest Amin is an employee of the University of California, Irvine and was a paid consultant to Bristol-Myers Squibb in connection with this study and the development of this manuscript. Keshishian is an employee of STATinMED Research, a paid consultant to Pfizer and Bristol-Myers Squibb in connection with this study and the development of this manuscript. Dina, Carda, Russ, Mardekian, and Baker are employees of Pfizer Inc., with ownership of stocks in Pfizer Inc. Dhamane, Nadkarni, and Rosenblatt are employees of BristolMyers Squibb Company, with ownership of stocks in Bristol-Myers Squibb Company. Yuce has no conflicts of interest.

Open Access This article is distributed under the terms of the Creative Commons Attribution 4.0 International License (http://creativeco mmons.org/licenses/by/4.0/), which permits unrestricted use, distribution, and reproduction in any medium, provided you give appropriate credit to the original author(s) and the source, provide a link to the Creative Commons license, and indicate if changes were made.

\section{References}

1. Chugh SS, Havmoeller R, Narayanan K et al (2014) Worldwide epidemiology of atrial fibrillation: a global burden of disease 2010 Study. Circulation 129(8):837-847

2. Colilla S, Crow A, Petkun W et al (2013) Estimates of current and future incidence and prevalence of atrial fibrillation in the U.S. adult population. Am J Cardiol 112(8):1142-1147

3. Naccarelli GV, Varker H, Lin J, Schulman KL (2009) Increasing prevalence of atrial fibrillation and flutter in the United States. Am J Cardiol 104(11):1534-1539

4. Björck S, Palaszewski B, Friberg L, Bergfeldt L (2013) Atrial fibrillation, stroke risk, and warfarin therapy revisited: a population-based study. Stroke 44(11):3103-3108

5. Lip GY, Nieuwlaat R, Pisters R, Lane DA, Crijns HJ (2010) Refining clinical risk stratification for predicting stroke and thromboembolism in atrial fibrillation using a novel risk factorbased approach: the euro heart survey on atrial fibrillation. Chest 137(2):263-272

6. January CT, Wann LS, Alpert JS et al (2014) 2014 AHA/ACC/ HRS guideline for the management of patients with atrial fibrillation: a report of the American College of Cardiology/American Heart Association Task Force on practice guidelines and the Heart Rhythm Society. Circulation 130(23):2071-2104

7. Sardar P, Chatterjee S, Chaudhari S, Lip GY (2014) New oral anticoagulants in elderly adults: evidence from a meta-analysis of randomized trials. J Am Geriatr Soc 62(5):857-864

8. Kim IS, Kim HJ, Kim TH et al (2018) Non-vitamin K antagonist oral anticoagulants have better efficacy and equivalent safety compared to warfarin in elderly patients with atrial fibrillation: a systematic review and meta-analysis. J Cardiol 72(2):105-112

9. Ruff CT, Giugliano RP, Braunwald E et al (2014) Comparison of the efficacy and safety of new oral anticoagulants with warfarin in patients with atrial fibrillation: a meta-analysis of randomised trials. Lancet 383(9921):955-962

10. Wehling M, Collins R, Gil VM et al (2017) Appropriateness of oral anticoagulants for the long-term treatment of atrial fibrillation in older people: results of an evidence-based review and international consensus validation process (OAC-FORTA 2016). Drugs Aging 34(7):499-507 
11. Amin A, Keshishian A, Trocio J et al (2017) Risk of stroke/ systemic embolism, major bleeding and associated costs in nonvalvular atrial fibrillation patients who initiated apixaban, dabigatran or rivaroxaban compared with warfarin in the United States Medicare population. Curr Med Res Opin 33(9):1595-1604

12. Centers for Medicare \& Medicaid Services (2015) Total Medicare Enrollment. CMS Chronic Conditions Data Warehouse. https:// www.cms.gov/Research-Statistics-Data-and-Systems/Statistics -Trends-and-Reports/CMSProgramStatistics/2015/Downloads/ MDCR_ENROLL_AB/2015_CPS_MDCR_ENROLL_AB_1. pdf. Accessed 17 July 2018

13. Chronic Condition Data Warehouse (2017) CCW White Paper: Medicare Claims Maturity. Chronic Condition Data Warehouse. https://www.ccwdata.org/web/guest/ccw-medicare-data-white -papers. Accessed 17 July 2018

14. Yao X, Abraham NS, Sangaralingham LR et al (2016) Effectiveness and safety of dabigatran, rivaroxaban, and apixaban versus warfarin in nonvalvular atrial fibrillation. J Am Heart Assoc 5(6): 003725

15. Thigpen JL, Dillon C, Forster KB et al (2015) Validity of international classification of disease codes to identify ischemic stroke and intracranial hemorrhage among individuals with associated diagnosis of atrial fibrillation. Circ Cardiovasc Qual Outcomes $8(1): 8-14$

16. Cunningham A, Stein CM, Chung CP et al (2011) An automated database case definition for serious bleeding related to oral anticoagulant use. Pharmacoepidemiol Drug Saf 20(6):560-566

17. Charlson ME, Pompei P, Ales KL, MacKenzie CR (1987) A new method of classifying prognostic comorbidity in longitudinal studies: development and validation. J Chronic Dis 40(5):373-383

18. Austin PC (2014) The use of propensity score methods with survival or time-to-event outcomes: reporting measures of effect similar to those used in randomized experiments. Stat Med 33(7):1242-1258

19. Austin PC (2009) Balance diagnostics for comparing the distribution of baseline covariates between treatment groups in propensity-score matched samples. Stat Med 28:3083-3107

20. Connolly SJ, Ezekowitz MD, Yusuf S et al (2009) Dabigatran versus warfarin in patients with atrial fibrillation. N Engl J Med 361(12):1139-1151

21. Patel MR, Mahaffey KW, Garg J et al (2011) Rivaroxaban versus warfarin in nonvalvular atrial fibrillation. N Engl J Med 365(10):883-891

22. Granger CB, Alexander JH, McMurray JJ et al (2011) Apixaban versus warfarin in patients with atrial fibrillation. N Engl J Med 365(11):981-992

23. Eikelboom JW, Wallentin L, Connolly SJ et al (2011) Risk of bleeding with 2 doses of dabigatran compared with warfarin in older and younger patients with atrial fibrillation: an analysis of the randomized evaluation of long-term anticoagulant therapy (RE-LY) trial. Circulation 123(21):2363-2372
24. Halperin JL, Hankey GJ, Wojdyla DM et al (2014) Efficacy and safety of rivaroxaban compared with warfarin among elderly patients with nonvalvular atrial fibrillation in the rivaroxaban once daily, oral, direct factor $\mathrm{Xa}$ inhibition compared with vitamin $\mathrm{K}$ antagonism for prevention of stroke and embolism trial in atrial fibrillation (ROCKET AF). Circulation 130(2):138-146

25. Halvorsen S, Atar D, Yang H et al (2014) Efficacy and safety of apixaban compared with warfarin according to age for stroke prevention in atrial fibrillation: observations from the ARISTOTLE trial. Eur Heart J 35(28):1864-1872

26. Graham DJ, Reichman ME, Wernecke M et al (2015) Cardiovascular, bleeding, and mortality risks in elderly Medicare patients treated with dabigatran or warfarin for non-valvular atrial fibrillation. Circulation 131(2):157-164

27. Deitelzweig S, Luo X, Gupta K et al (2017) Comparison of effectiveness and safety of treatment with apixaban vs. other oral anticoagulants among elderly nonvalvular atrial fibrillation patients. Curr Med Res Opin 33(10):1745-1754

28. Avgil-Tsadok M, Jackevicius CA, Essebag V et al (2016) Dabigatran use in elderly patients with atrial fibrillation. Thromb Haemost 115(1):152-160

29. Coleman CI, Weeda ER, Nguyen E, Bunz TJ, Sood NA (2018) Effectiveness and safety of rivaroxaban vs. warfarin in patients $80+$ years of age with non-valvular atrial fibrillation. Eur Heart J Qual Care Clin Outcomes 4(4):328-329

30. Seeger JD, Bykov K, Bartels DB et al (2015) Safety and effectiveness of dabigatran and warfarin in routine care of patients with atrial fibrillation. Thromb Haemost 114(6):1277-1289

31. Norby FL, Bengtson LG, Lutsey PL et al (2017) Comparative effectiveness of rivaroxaban versus warfarin or dabigatran for the treatment of patients with non-valvular atrial fibrillation. BMC Cardiovasc Disord 17(1):238

32. Li XS, Deitelzweig S, Keshishian A et al (2017) Effectiveness and safety of apixaban versus warfarin in non-valvular atrial fibrillation patients in "real-world" clinical practice. a propensity-matched analysis of 76,940 patients. Thromb Haemost 117(6):1072-1082

33. Patel PA, Zhao X, Fonarow GC et al (2015) Novel oral anticoagulant use among patients with atrial fibrillation hospitalized with ischemic stroke or transient ischemic attack. Circ Cardiovasc Qual Outcomes 8(4):383-392

34. Di Minno MN, Ambrosino P, Dentali F (2017) Safety of warfarin in "high-risk" populations: a meta-analysis of randomized and controlled trials. Thromb Res 150:1-7

Publisher's Note Springer Nature remains neutral with regard to jurisdictional claims in published maps and institutional affiliations. 